\title{
Probing Thermo-Mechanical Properties of Polymers for High Resolution Tg Mapping.
}

\author{
M.P. Nikiforov, ${ }^{*}$ L.T. Germinario, ${ }^{* *}$ S. Gam, ${ }^{* * *}$ R.C. Composto, ${ }^{* * *}$ S. Jesse, ${ }^{*}$ S.V. Kalinin*
}

* Center for Nanophase Materials Science, Oak Ridge National Laboratory, PO Box 2008, Oak Ridge, TN 37831-6487

** LG Analytical, Kingsport, TN

*** Department of the Materials Science and Engineering, University of Pennsylvania, Philadelphia, PA, 19104

Polymer materials are used in many industries because of the tunability of their properties. Large variations in the materials' properties are often achieved by blending different polymers together in a homogeneous mixture. However, blends of polymers tend to phase separate causing failure of the devices, in which such materials were used. Thermo-mechanical analysis was successfully used to determine usable temperature range for these materials. In order to understand mechanisms involved in phase separation of bends local analysis of thermo-mechanical properties is desirable. Currently, several scanning probe microscopy based techniques for local thermal analysis exist (Transition Temperature Microscopy (TTM) [1], Scanning Thermal Expansion Microscopy (SThEM) [2], Thermally Assisted Atomic Force Acoustic Microscopy (TA-AFAM) [3], ZTherm [4] and Band Excitation-NanoTA (BE-NanoTA) [5]). All these techniques are based on characterization of the contact properties. Only two techniques (ZTherm and BE-NanoTA) are capable of providing complete information about contact properties, such as contact stiffness, contact damping coefficient etc.

This talk will be focused on BE-NanoTA method, which is the most versatile technique for contact characterization of all the techniques presented above. It allows direct determination of the mechanical properties of the tip-surface junction as a function of temperature by measuring mechanical response in the band of frequencies and comparing it with the excitation signal spanning the same band of frequencies. There are two implementations for this method. The first one is the modification of the atomic force acoustic microscopy (AFAM) technique by mechanical excitation and oscillation detection over a broad frequency range (band excitation (BE) technique), and by using the heated tip probe (BE-AFAM). The second one is the use of the heated tip and thermal expansion of the material combined in conjunction with the BE technique (BE-SThEM). The BE technique used in the BE-NanoTA method provides direct measurement of the four parameters of system resonance (resonance amplitude, resonance frequency, quality factor and phase).

Furthermore, a protocol that maintains a constant tip/surface pressure and reproducible contact area during a temperature sweep has been reported, effectively extending the Oliver-Pharr method for NI to local thermal analysis. This method also provides measurements of mechanical properties as well as glass transition temperatures with sub- $100 \mathrm{~nm}$ resolution. Specialized probes with a heater at the end are required for both implementations of this technique. The AFAM-based implementation of the method requires the use of the transducer to induce mechanical oscillations of the sample.

The applications of the BE-NanoTA technique for local measurements of Young's modulus on PET, [6] creation of glass transition temperature maps on phase separated SAN:PMMA blend as well as 
contact mechanics models necessary for quantitative interpretation of the measurements will be discussed. [7]

References:

[1] Anasys Instruments Co (www.anasysinstruments.com).

[2] A. Hammiche et al., Journal of Microscopy-Oxford 2000, 199, 180.

[3] F. Oulevey et al., Review of Scientific Instruments 1998, 69, (5), 2085.

[4] Asylum Research Co (http://www.asylumresearch.com/Products/Ztherm/Ztherm.shtml).

[5] M.P. Nikiforov et al., Nanotechnology 2009, 20, (39), 395709.

[6] S. Jesse et al., Appl. Phys. Lett. 2008, 93, (7), 073104

[7] A portion of this research at the Oak Ridge National Laboratory's Center for Nanophase Materials Sciences was sponsored by the Scientific User Facilities Division, Office of Basic Energy Sciences, U. S. Department of Energy. The work by RJC and SG was supported by the Nano / Bio Interface Center at the University of Pennsylvania and the U.S. National Science Foundation (NSF) under grant number DMR04-25780 as well as the World Materials Network (NSF) DMR09-08449. Partial support was provided by NSF under grant number, DMR0907493. 